\title{
Combination of structural MRI, functional MRI and brain PET-CT provide more diagnostic and prognostic value in patients of cerebellar ataxia associated with anti-Tr/ DNER: a case report
}

Sisi Shen ${ }^{1+}$, Wenyu Liu ${ }^{1+}$, Ming Zhou², Ruiyi Yang ${ }^{2}$, Jinmei Li ${ }^{1 *}$ (D) and Dong Zhou ${ }^{1 *}$

\begin{abstract}
Background: Brain magnetic resonance imaging (MRI) rarely reveals structural changes in patients with suspected anti-Tr/DNER encephalitis and thus provides very limited information. Here, we combined structural MRI, functional $\mathrm{MRI}$, and positron emission tomography-computed tomography (PET-CT) findings to characterize this rare disorder in a patient.

Case presentation: A 43-year-old woman presented with progressive cerebellar ataxia, memory impairment, anxiety, and depression. Anti-Tr antibodies were detected in both her serum (1:10) and cerebrospinal fluid (1:10). A diagnosis of anti-Tr-positive autoimmune cerebellar ataxia was established. The patient's symptoms were worse, but her brain MRI was normal. Meanwhile, voxel-based morphometry analysis showed bilateral reduced cerebellar volume, especially in the posterior lobe and uvula of the cerebellum and the middle of the left temporal lobe compared with 6 sex- and age-matched healthy subjects ( 6 females, $43 \pm 2$ years; $p<0.05$ ). Using seed-based functional connectivity analysis, decreased connectivity between the posterior cingulate cortex/precuneus and left frontal lobe compared to the control group $(p<0.05)$ was detected. PET-CT revealed bilateral hypometabolism in the cerebellum and relative hypermetabolism in the cerebellar vermis and bilateral frontal lobe, but no malignant changes.
\end{abstract}

Conclusions: A combination of structural MRI, functional MRI, and brain PET-CT has higher diagnostic and prognostic value than conventional MRI in patients with suspected anti-Tr/DNER encephalitis.

\footnotetext{
* Correspondence: jinmeili-neuro@qq.com; zhoudong66@yahoo.de

†'Sisi Shen and Wenyu Liu have contributed equally to this paper and should be considered co-first authors.

'Department of Neurology, West China Hospital, Sichuan University, No. 37 GuoXue Alley, 610041 Chengdu, Sichuan, China

Full list of author information is available at the end of the article
}

(c) The Author(s). 2021 Open Access This article is licensed under a Creative Commons Attribution 4.0 International License, which permits use, sharing, adaptation, distribution and reproduction in any medium or format, as long as you give appropriate credit to the original author(s) and the source, provide a link to the Creative Commons licence, and indicate if changes were made. The images or other third party material in this article are included in the article's Creative Commons licence, unless indicated otherwise in a credit line to the material. If material is not included in the article's Creative Commons licence and your intended use is not permitted by statutory regulation or exceeds the permitted use, you will need to obtain permission directly from the copyright holder. To view a copy of this licence, visit http://creativecommons.org/licenses/by/4.0/ The Creative Commons Public Domain Dedication waiver (http://creativecommons.org/publicdomain/zero/1.0/) applies to the data made available in this article, unless otherwise stated in a credit line to the data. 


\section{Background}

Cerebellar ataxia associated with anti-Tr/DNER (Delta/ Notch-like epidermal growth factor-related receptor) autoantibodies is a rare autoimmune disease characterized by progressively acute or sub-acute severe cerebellar ataxia that eventually disables affected patients [1-5]. Progression of this disorder is often irreversible, which is consistent with the total loss of cerebellar Purkinje cells observed at autopsy [4]. A full understanding and early diagnosis of this disease is crucial, as prompt treatment can prevent disability [6]. The major feature of this disorder is severe cerebellar ataxia, which highlights abnormalities of the cerebellum. However, patients often present with other symptoms, such as extensor plantar response [7], retrobulbar optic neuropathy [7], encephalopathy [4], sensory neuropathy and limbic encephalitis [4], which is indicative of the involvement of areas outside the cerebellum. Detecting areas affected in this rare disorder by morphological examination is important, because exploring the associations between affected areas and clinical manifestations may help clarify the pathophysiological mechanisms and predict prognosis of this disease.

However, initial evaluations using conventional brain magnetic resonance imaging (MRI) rarely reveals structural changes $[1,5]$. Even when changes are present, they are often subtle or nonspecific, resulting in MRIs providing very limited information. Voxel-based morphometry (VBM), which is an MRI processing technique that can detect regional morphological changes throughout the brain, resting state functional MRI (fMRI), which is an emerging functional imaging technique that analyzes spontaneous fluctuations in the blood oxygen leveldependent (BOLD) signal to assess functional connectivity (FC) of remote brain areas, and positron emission tomography-computed tomography (PET-CT) have been successfully used to detect structural and functional changes in various nervous system diseases. Here, we hypothesize that multimodal imaging analyses may also reveal structural and functional changes in the brains of patients with anti-Tr/DNER cerebellar ataxia, increasing the pathophysiological and prognostic value of these assessments. In this study, we combined MRI with VBM, FC, and PET-CT to assess a patient and characterize this rare disorder.

\section{Case presentation}

A 43-year-old woman presented with dizziness for 3 months along with worsening dysarthria and ataxia for 1 month. Apart from severe cerebellar ataxia, she also complained of depression for 2 months, as well as memory loss and blurred vision for 2 weeks. Physical examination showed speech dysarthria and bilateral horizontal gaze-evoked nystagmus that was more obviously towards the right. Finger-nose and heel-shin tests revealed severe ataxia, which had rendered the patient bedridden. Laboratory findings, including complete blood cell count and biochemical, metabolic, infectious, immunologic, and serologic tests, were normal. Cerebrospinal fluid and conventional brain MRI examination were unremarkable. The patient had a Mini Mental Status Examination (MMSE) score of 27 and a Montreal Cognitive Assessment (MOCA) total score of 21. She experienced impairment of short-term memory (2/5), visuospatial functions (1/5), and attention (4/6). The Hamilton Anxiety Scale (HAMA) and Hamilton Depression Scale (HAMA) revealed mild anxiety (15) and moderate depression (23). Anti-Tr antibodies were detected in both her serum (1:10) and cerebrospinal fluid (1:10). Due to the strong association of anti- $\mathrm{Tr}$ with malignancy, whole-body contrast computed tomography, ultrasounds of thyroid, breast, and reproductive organs, and bone marrow aspiration were performed for further investigation. However, no malignant changes were found. A diagnosis of anti-Tr positive autoimmune cerebellar ataxia in the absence of malignancy was established and the patient received immune therapy successively. Patient therapy consisted of steroid pulse therapy (5 days of $1 \mathrm{~g} / \mathrm{d}$ intravenous methylprednisolone sodium succinate, and then $60 \mathrm{mg} / \mathrm{d}$ prednisone) followed by intravenous immunoglobulin $(0.4 \mathrm{~g} / \mathrm{kg}$ per day for 5 days $)$. After intravenous therapy, the patient was discharged from the hospital and underwent rehabilitation at home with continual prednisone treatment that was decreased weekly by $5 \mathrm{mg}$. Patient symptoms and treatment were shown in Fig. $1 \mathrm{~A}$.

Upon her re-admission 1 month later, the patient's major complaint was worsening of the ataxia and an increased cognitive-emotional deficit. In recent years, increasing evidence for a role for the cerebellum in emotion and cognition has emerged [8-12] and the patient was identified as having cerebellar cognitive affective syndrome [13]. The default mode network (DMN) has become the primary and most popular target of resting state networks and is thought to be involved in advanced cognitive functions and emotion [14-16]. Recent fMRI studies have linked activity to cognition and explored how FC is altered in several diseases. The disrupted connectivity within the DMN and remote interregional connectivity related to hubs of DMN have been widely reported in terms of changes related to cognitive deficits. The posterior cingulate cortex (PCC)/precuneus is a pivotal hub in the DMN [17] and also has been linked to many cognitive-emotional disorders [15, $16,18]$. Therefore, to further assess the structural and functional abnormalities and reveal the mechanism underlying the cognitive-emotional deficit in this patient, VBM, PCC and cerebellum seed-based FC analysis were 

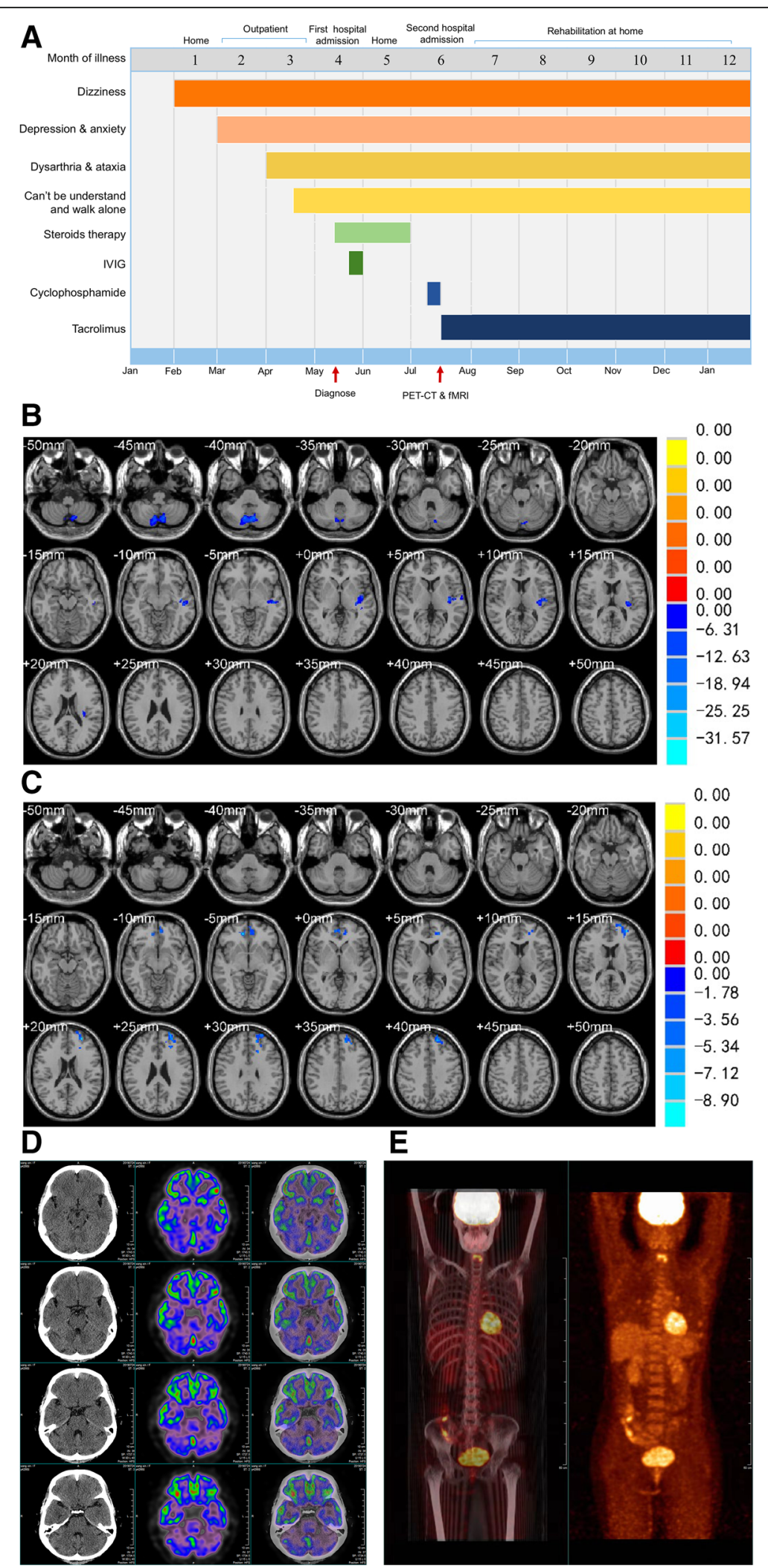

Fig. 1 (See legend on next page.) 
(See figure on previous page.)

Fig. 1 Clinical and imaging features of cerebellar ataxia patient associated with anti-Tr/DNER antibodies. A: The symptoms and treatment measures of the patient; B: VBM analysis showed reduced cerebellar volume bilaterally, especially in the posterior lobe and uvula of cerebellum, and the middle of the left temporal lobe compared with controls; $\mathbf{C}$ : The seed-based FC investigation showed lower connectivity between posterior cingulate cortex (PCC)/precuneus and left frontal lobe than that of the control group; D: Brain PET-CT showed obvious decreased fluorodeoxyglucose (FGD) uptake in bilateral cerebellum; E: whole-body PET-CT showed no malignant changes were found

performed at the whole-brain level in addition to PETCT. Acquisition sequences of sMRI and fMRI were seen in supplementary materials.

VBM analysis showed bilateral reductions in cerebellar volume, especially in the posterior lobe and uvula of cerebellum and the middle of the left temporal lobe in comparison with 6 sex- and age-matched healthy subjects (6 females, $43 \pm 2$ years; $p<0.05$; Fig. 1B). Seedbased FC assessment identified reduced connectivity between the PCC/precuneus and left frontal lobe compared to the control group $(p<0.05$; Fig. 1C). PET-CT revealed bilateral hypometabolism of the cerebellum and relative hypermetabolism of the cerebellar vermis and bilateral frontal lobe (Fig. 1D), but no malignant changes (Fig. 1E). An intravenous of injection of cyclophosphamide was offered at $0.4 \mathrm{~g} / \mathrm{d}$ for 3 days, followed by Tacrolimus as a long-term immunosuppressive treatment. Unfortunately, the patient failed to improve.

At one-year follow up, the patient repeated the wholebody contrast PET-CT, ultrasounds of thyroid, breasts, and reproductive organs, and bone marrow biopsy with no evidence of malignancy. At this time, her symptoms have not improved.

\section{Discussion and conclusions}

We report a confirmed case of anti- $\mathrm{Tr}$ positive autoimmune cerebellar ataxia with no signs of malignancy at 1 year follow up. Studies show that $84.6-94.7 \%$ of patients with this disease also present with Hodgkin's lymphoma with a median time between diagnosis of cerebellar ataxia and Hodgkin's lymphoma or any other tumor type being 3.5 months (ranging from 0 to 24 months) $[2,4,5]$. No tumors have been found in our patient, suggesting that, in a few instances, the origin of the anti-Tr antibodies is not a tumor but another unknown cause, such as a para-infectious origin [1]. It is important to note that the follow-up period may have been too short to confidently rule out malignancy for this patient.

Our study illustrates multimodality imaging can be more sensitive than conventional MRI in detection of brain damage and may aid in explaining clinicradiological discrepancies. In this patient, bilateral reductions in volume and obvious hypometabolism in the cerebellum were observed, which is consistent with her irreversible severe cerebellar ataxia. A postpartum study of patients with anti-Tr/DNER cerebellar ataxia found strong anatomical evidence in the form of the total loss of cerebellar Purkinje cells [4]. Of note, relative hypermetabolism in the cerebellar vermis and bilateral frontal lobe was also observed in our patient, which might be indicative of underlying inflammation [19]. Regions with abnormal hypometabolism and hypermetabolism as identified by PET-CT are also observed in autoimmune encephalitis [19] and paraneoplastic cerebellar degeneration [20]. Reduced middle of the left temporal lobe volume and lower connectivity between the PCC and left frontal lobe can explain patient's cognitive-emotional deficit. These structures are all parts of the DMN, which has been linked to cognitive-emotional processing [17]. Grimm and Simone et al. investigated neural activity in the DMN during different emotional tasks in healthy subjects and patients with major depressive disorder in an event-related fMRI design. They found decreased negative BOLD responses in the PCC and ventromedial prefrontal cortex in patients with major depressive disorder that correlated with depression severity and feelings of hopelessness [15]. The PCC and ventromedial prefrontal cortex have also been shown to be involved in short-term memory, visuospatial functions, and attention using a number of fMRI studies [14, 18, 21]. Involvement of extra-cerebellar regions is also supported by neuroanatomy studies. In rats, anti- $\operatorname{Tr}$ antibody immunoreactivity was not confined to the Purkinje cells of the cerebellum but was also observed in the plexiform layers of the hippocampus and neocortex [22, 23].

The cerebellum may also play a role in cognitiveemotional deficits in patient. Anatomically, there are extensive anatomical connections between the cerebellum and associated paralimbic areas, such as the temporoparietal junction, lateral temporal cortex, PCC, inferior frontal gyrus, amygdala, and insula [24, 25]. Most of these are part of the DMN described above and involved in processing of cognition and emotions. Additional insight was derived from VBM studies $[9,26]$. For example, Olivito et al. assessed the relationship between cerebellar gray matter (GM) atrophy and neuropsychological scores in patients with spinocerebellar ataxia 2 using VBM and found GM loss in the cognitive posterior lobules (VI, Crus I, Crus II, VIIB, and IX) that correlated with visuospatial, verbal memory, and executive tasks [26]. Further evidence has been provided by RS- 
fMRI studies that showed altered FC between cerebellar and cerebrum regions, which are known to be related to cognition and emotion, in patients with spinocerebellar ataxia 2, depression, and Alzheimer's disease [9, 27-30]. In our patient, VBM analysis revealed atrophy in both the DMN structure and cerebellum. FC analysis demonstrated decreased connectivity in the DMN. Based on the studies mentioned above, we suggest the cognitiveemotional deficits in our patient were caused by disfunction of the cerebellum and DMN.

Notably, the reduced left frontal lobe-precuneus connectivity seen in our patient is nonspecific. Recent work has shown impairment of motor and cognitive cerebellar-neocortical networks in chronic ataxias, such as spinocerebellar ataxia 17 [31]. Similarly, other groups have recently outlined deficits in cerebellar-neocortical networks and cerebellar-striatal connectivity in patients with multiple system atrophy (MSA) [32] and overactivity in the olivary-cerebellar-thalamic network in patients with an essential tremor [33]. Here, we give the first insight into cerebellar-neocortical network functionality in anti-Tr positive autoimmune cerebellar ataxia. However, caution is needed when interpreting our results as this study consisted of only one patient. Our findings should be evaluated using larger cohort studies.

In conclusion, we report a confirmed case of anti-Tr positive autoimmune cerebellar ataxia with no tumor signs at 1 year follow up. A combination of structural MRI, functional MRI, and brain PET-CT has diagnostic and prognostic value in patients with suspected anti- $\mathrm{Tr} /$ DNER encephalitis. Disfunction of the cerebellum and DMN may contribute to the cognitive-emotional deficits seen in this disorder.

\section{Abbreviations}

DNER: Delta/Notch-like epidermal growth factor-related receptor; MRI: Magnetic resonance imaging; VBM: Voxel-based morphometry; RSfMRI: Resting state functional magnetic resonance imaging; BOLD: Blood oxygen level-dependent; FC: Functional connectivity; PET-CT: Positron emission tomography-computed tomography; CSF: Cerebrospinal fluid; MMSE: Mini Mental Status Examination; MOCA: Montreal Cognitive Assessment; HAMA: Hamilton Anxiety Scale; HAMD: Hamilton Depression Scale; DMN: Default mode network; PCC: Posterior cingulate cortex; GM: Gray matter; MSA: Multiple system atrophy

\section{Supplementary Information}

The online version contains supplementary material available at https://doi. org/10.1186/s12883-021-02403-5.

\section{Additional file 1.}

\section{Acknowledgements}

Not applicable.

\section{Authors' contributions}

Data acquisition: SSS, WYL, MZ and RYY; data interpretation: SSS, WYL; writing —original draft preparation: SSS; writing —review and editing: WYL, MZ and RYY; design and conceptualization: JML, DZ. All authors have read and approved this manuscript.
Funding

None.

Availability of data and materials

All data used in this study are available from the corresponding author on reasonable request.

\section{Declarations}

Ethics approval and consent to participate

This study was approved by the ethics committee of West China Hospital.

\section{Consent for publication}

Written informed consent was obtained from the patient for publication of this case report and any accompanying images.

\section{Competing interests}

None disclosed.

\section{Author details}

'Department of Neurology, West China Hospital, Sichuan University, No. 37 GuoXue Alley, 610041 Chengdu, Sichuan, China. ${ }^{2}$ Department of Radiology, the Third Hospital of Mianyang, Sichuan Mental Health Center, No. 190 East of Jiannan Road, 621000 Mianyang, Sichuan, China.

Received: 3 October 2020 Accepted: 16 September 2021

Published online: 24 September 2021

\section{References}

1. de Graaff E, Maat P, Hulsenboom E, van den Berg R, van den Bent M, Demmers J, et al. Identification of delta/notch-like epidermal growth factorrelated receptor as the $\operatorname{Tr}$ antigen in paraneoplastic cerebellar degeneration Ann Neurol. 2012;71(6):815-24.

2. Probst C, Komorowski L, de Graaff E, van Coevorden-Hameete M, Rogemond V, Honnorat J, et al. Standardized test for anti-Tr/DNER in patients with paraneoplastic cerebellar degeneration. Neurol Neuroimmunol Neuroinflamm. 2015;2(2):e68. https://doi.org/10.1212/nxi.0000000000000068.

3. Briani C, Vitaliani R, Grisold W, Honnorat J, Graus F, Antoine JC, et al. Spectrum of paraneoplastic disease associated with lymphoma. Neurology. 2011;76:8.

4. Bernal F, Shams'ili S, Rojas I, Sanchez-Valle R, Saiz A, Dalmau J, et al. Anti-Tr antibodies as markers of paraneoplastic cerebellar degeneration and Hodgkin's disease. Neurology. 2003:60(2):230-4.

5. Jarius S, Wildemann B. 'Medusa head ataxia': the expanding spectrum of Purkinje cell antibodies in autoimmune cerebellar ataxia. Part 3: Anti-Yo/ CDR2, anti-Nb/AP3B2, PCA-2, anti-Tr/DNER, other antibodies, diagnostic pitfalls, summary and outlook. J Neuroinflammation. 2015;12:168. https://doi. org/10.1186/s12974-015-0358-9.

6. Razzak A, Shields M, Allsup D. Autoimmune paraneoplastic cerebellar degeneration secondary to a composite lymphoma. Leuk Lymphoma. 2007; 48(7):1456-8.

7. Graus F, Dalmau J, Valldeoriola F, Ferrer I, Rene R, Marin C, et al. Immunological characterization of a neuronal antibody (anti-Tr) associated with paraneoplastic cerebellar degeneration and Hodgkin's disease. J Neuroimmunol. 1997;74(1-2):55-61. https://doi.org/10.1016/s0165-572 8(96)00205-6.

8. Clausi S, Lupo M, Olivito G, Siciliano L, Contento MP, Aloise F, et al. Depression disorder in patients with cerebellar damage: awareness of the mood state. J Affect Disord. 2019;245:386-93. https://doi.org/10.1016/j.jad.2 018.11.029.

9. Lupo M, Olivito G, lacobacci C, Clausi S, Romano S, Masciullo M, et al. The cerebellar topography of attention sub-components in spinocerebellar ataxia type 2. Cortex. 2018;108:35-49. https://doi.org/10.1016/j.cortex.2018. 07.011.

10. Lupo M, Olivito G, Siciliano L, Masciullo M, Molinari M, Cercignani M, et al. Evidence of Cerebellar Involvement in the Onset of a Manic State. Front Neurol. 2018:9:774. https://doi.org/10.3389/fneur.2018.00774.

11. Clausi S, lacobacci C, Lupo M, Olivito G, Molinari M, Leggio M. The role of the cerebellum in unconscious and conscious processing of emotions: review. Appl Sci. 2017;7(5):521. 
12. Tedesco AM, Chiricozzi FR, Clausi S, Lupo M, Molinari M, Leggio MG. The cerebellar cognitive profile. Brain. 2011;134(12):3672-86. https://doi.org/10.1 093/brain/awr266.

13. Schmahmann JD, Sherman JC. The cerebellar cognitive affective syndrome. Brain. 1998;121(Pt 4):561-79. https://doi.org/10.1093/brain/121.4.561.

14. Geday J, Gjedde A. Attention, emotion, and deactivation of default activity in inferior medial prefrontal cortex. Brain Cogn. 2009;69(2):344-52. https:// doi.org/10.1016/j.bandc.2008.08.009.

15. Grimm S, Boesiger P, Beck J, Schuepbach D, Bermpohl F, Walter M, et al. Altered Negative BOLD responses in the default-mode network during emotion processing in depressed subjects. Neuropsychopharmacology. 2009;34(4):932-43. https://doi.org/10.1038/npp.2008.81.

16. Shim G, Oh JS, Jung WH, Jang JH, Choi C-H, Kim E, et al. Altered restingstate connectivity in subjects at ultra-high risk for psychosis: an fMRI study. \#N/A. 2010;6(1):58. https://doi.org/10.1186/1744-9081-6-58.

17. Sreenivas S, Boehm SG, Linden DEJ. Emotional faces and the default mode network. Neurosci Lett. 2012;506(2):229-34. https://doi.org/10.1016/j.neulet.2 011.11.012.

18. Zarifkar P, Kim J, La C, Zhang K, YorkWilliams S, Levine TF, et al. Cognitive impairment in Parkinson's disease is associated with default mode network subsystem connectivity and cerebrospinal fluid A $\beta$. Parkinsonism Relat Disord. 2021;83:71-8. https://doi.org/10.1016/j.parkreldis.2021.01.002.

19. Probasco JC, Solnes L, Nalluri A, Cohen J, Jones KM, Zan E, et al. Abnormal brain metabolism on FDG-PET/CT is a common early finding in autoimmune encephalitis. Neurol Neuroimmunol Neuroinflamm. 2017:4(4): e352. https://doi.org/10.1212/nxi.0000000000000352.

20. Abdulaziz ATA, Yu XQ, Zhang L, Jiang XY, Zhou D, Li JM. Paraneoplastic cerebellar degeneration associated with cerebellar hypermetabolism: case report. Med (Baltim). 2018;97(24):e10717. https://doi.org/10.1097/md. 0000000000010717

21. Lehmann M, Madison CM, Ghosh PM, Seeley WW, Mormino E, Greicius MD, et al. Intrinsic connectivity networks in healthy subjects explain clinical variability in Alzheimer's disease. Proc Natl Acad Sci U S A. 2013;110(28): 11606-11. https://doi.org/10.1073/pnas.1221536110.

22. Eiraku M, Hirata Y, Takeshima H, Hirano T, Kengaku M. Delta/Notch-like Epidermal Growth Factor (EGF)-related Receptor, a Novel EGF-like Repeatcontaining Protein Targeted to Dendrites of Developing and Adult Central Nervous System Neurons*. J Biol Chem. 2002;277(28):25400-7. https://doi. org/10.1074/jbc.M110793200.

23. Graus F, Gultekin SH, Ferrer I, Reiriz J, Alberch J, Dalmau J. Localization of the neuronal antigen recognized by anti-Tr antibodies from patients with paraneoplastic cerebellar degeneration and Hodgkin's disease in the rat nervous system. Acta Neuropathol. 1998;96(1):1-7.

24. Mar RA. The neural bases of social cognition and story comprehension. Annu Rev Psychol. 2011;62:103-34. https://doi.org/10.1146/annurevpsych-120709-145406.

25. Calder AJ, Lawrence AD, Young AW. Neuropsychology of fear and loathing. \#N/A. 2001;2(5):352-63. https://doi.org/10.1038/35072584.

26. Olivito G, Lupo M, lacobacci C, Clausi S, Romano S, Masciullo M, et al. Structural cerebellar correlates of cognitive functions in spinocerebellar ataxia type 2. J Neurol. 2018;265(3):597-606. https://doi.org/10.1007/s00415018-8738-6

27. Wei Q, Ji Y, Bai T, Zu M, Guo Y, Mo Y, et al. Enhanced cerebro-cerebellar functional connectivity reverses cognitive impairment following electroconvulsive therapy in major depressive disorder. Brain Imaging Behav. 2021;15(2):798-806. https://doi.org/10.1007/s11682-020-00290-x.

28. Guo W, Liu F, Xue Z, Gao K, Liu Z, Xiao C, et al. Abnormal resting-state cerebellar-cerebral functional connectivity in treatment-resistant depression and treatment sensitive depression. Prog Neuropsychopharmacol Biol Psychiatry. 2013;44:51-7. https://doi.org/10.1016/j.pnpbp.2013.01.010.

29. Pagen $L H G$, van de Ven VG, Gronenschild E, Priovoulos N, Verhey FRJ, Jacobs HIL. Contributions of Cerebro-Cerebellar Default Mode Connectivity Patterns to Memory Performance in Mild Cognitive Impairment. \#N/A. 2020; 75(2):633-47. https://doi.org/10.3233/jad-191127.

30. Olivito G, Serra L, Marra C, Di Domenico C, Caltagirone C, Toniolo S, et al. Cerebellar dentate nucleus functional connectivity with cerebral cortex in Alzheimer's disease and memory: a seed-based approach. Neurobiol Aging. 2020:89:32-40. https://doi.org/10.1016/j.neurobiolaging.2019.10.026.

31. Reetz K, Dogan I, Rolfs A, Binkofski F, Schulz JB, Laird AR, et al. Investigating function and connectivity of morphometric findings-exemplified on cerebellar atrophy in spinocerebellar ataxia 17 (SCA17). Neurolmage. 2012; 62(3):1354-66. https://doi.org/10.1016/j.neuroimage.2012.05.058.

32. Baggio HC, Abos A, Segura B, Campabadal A, Uribe C, Giraldo DM, et al. Cerebellar resting-state functional connectivity in Parkinson's disease and multiple system atrophy: Characterization of abnormalities and potential for differential diagnosis at the single-patient level. Neuroimage Clin. 2019;22: 101720. https://doi.org/10.1016/j.nicl.2019.101720.

33. Fang W, Lv F, Luo T, Cheng O, Liao W, Sheng K, et al. Abnormal regional homogeneity in patients with essential tremor revealed by resting-state functional MRI. PLoS One. 2013;8(7):e69199. https://doi.org/10.1371/journal. pone.0069199.

\section{Publisher's Note}

Springer Nature remains neutral with regard to jurisdictional claims in published maps and institutional affiliations.
Ready to submit your research? Choose BMC and benefit from:

- fast, convenient online submission

- thorough peer review by experienced researchers in your field

- rapid publication on acceptance

- support for research data, including large and complex data types

- gold Open Access which fosters wider collaboration and increased citations

- maximum visibility for your research: over $100 \mathrm{M}$ website views per year

At BMC, research is always in progress.

Learn more biomedcentral.com/submissions 